\title{
Tackling inequalities in obesity: a protocol for a systematic review of the effectiveness of public health interventions at reducing socioeconomic inequalities in obesity among adults
}

Clare L Bambra ${ }^{1}$, Frances C Hillier ${ }^{1 *}$, Helen J Moore ${ }^{2}$, Joanne-Marie Cairns-Nagi ${ }^{1}$ and Carolyn D Summerbell ${ }^{2}$

\begin{abstract}
Background: Socioeconomic inequalities in obesity and associated risk factors for obesity are widening throughout developed countries worldwide. Tackling obesity is high on the public health agenda both in the United Kingdom and internationally. However, what works in terms of interventions that are able to reduce inequalities in obesity is lacking.
\end{abstract}

Methods/Design: The review will examine public health interventions at the individual, community and societal level that might reduce inequalities in obesity among adults aged 18 years and over, in any setting and in any country. The following electronic databases will be searched: MEDLINE, EMBASE, CINAHL, PsycINFO, Social Science Citation Index, ASSIA, IBSS, Sociological Abstracts, and the NHS Economic Evaluation Database. Database searches will be supplemented with website and gray literature searches. No studies will be excluded based on language, country or publication date. Randomized and non-randomized controlled trials, prospective and retrospective cohort studies (with/without control groups) and prospective repeat cross-sectional studies (with/without control groups) that have a primary outcome that is a proxy for body fatness and have examined differential effects with regard to socioeconomic status (education, income, occupation, social class, deprivation, poverty) or where the intervention has been targeted specifically at disadvantaged groups or deprived areas will be included. Study inclusion, data extraction and quality appraisal will be conducted by two reviewers. Meta-analysis and narrative synthesis will be conducted. The main analysis will examine the effects of 1) individual, 2) community and 3) societal level public health interventions on socioeconomic inequalities in adult obesity. Interventions will be characterized by their level of action and their approach to tackling inequalities. Contextual information on how such public health interventions are organized, implemented and delivered will also be examined.

Discussion: The review will provide evidence, and reveal any gaps in the evidence base, of public health strategies which reduce and prevent inequalities in the prevalence of obesity in adults and provide information on the organization, implementation and delivery of such interventions.

Trial registration: PROSPERO registration number: CRD42013003612

Keywords: Obesity, Socioeconomic inequalities, Public health, Adults

\footnotetext{
* Correspondence: frances.hillier@durham.ac.uk

${ }^{1}$ Department of Geography, Wolfson Research Institute, Durham University

Queen's Campus, University Boulevard, Stockton-on-Tees TS17 6BH, UK

Full list of author information is available at the end of the article
} 


\section{Background}

There is a strong and growing evidence base that shows the impact of obesity on short and long-term functioning, health and wellbeing [1]. Obesity has been linked to chronic diseases such as diabetes, coronary heart disease, stroke, hypertension, osteoarthritis and certain forms of cancer [2] thus demonstrating the significance of obesity for health and wellbeing. Internationally, adult obesity rates continue to rise as seen in the latest Organisation for Economic Co-operation and Development (OECD) figures. According to these figures, more than half (50.3\%) of the OECD member countries have an adult population who were classified as obese or overweight [3]. This staggering figure shows that adult obesity rates have doubled, and in some cases tripled, since 1980. It has been estimated that based on healthcare costs in the United Kingdom (UK) the burden of diseases associated with obesity will cost approximately $£ 10$ billion per year by 2050 [4]. Consequently, the number of early deaths, long-term incapacity and reduction in quality of life will escalate. Taking England as an example, recent figures show that just over a quarter of adults (26\% of both men and women over the age of 16) were classified as obese [5]. Therefore, tackling obesity is rightly highlighted as one of the major contemporary public health concerns and vital for addressing socioeconomic inequalities in poor health.

\section{Inequalities in obesity}

Studies have found that in most European countries, the United States and Australia socioeconomic inequalities in obesity and associated risk factors for obesity are widening $[1,3,4,6,7]$. A global assessment of these socioeconomic inequalities in obesity is important in order to prevent and reduce the development of chronic diseases.

In the UK, as is the case in most other high income countries, obesity is more prevalent in the lowest income quintile [8]. There is little evidence of change over time [9]. Mackenbach and colleagues' (2008) research shows that socioeconomic inequalities in obesity are larger among women, particularly in the southern region of Europe, whereby in some countries education-related inequalities in obesity are almost four times higher for those with the least education [10]. Moreover, it has been found that there is also a clear socioeconomic gradient in body mass index (BMI) for residents living in more deprived neighborhoods, independent of individual socioeconomic position [11]. Geographical inequalities are also evident in England, with hotspots in the North East, Yorkshire and Humber, and the East and West Midlands [9] and a clear north-south divide [5]. Longitudinal analyses also suggest that the social gradient in obesity is associated with the accumulation of disad- vantage throughout the life course contributed to widening inequalities in obesity in adulthood and this trend is more pronounced for women [8].

\section{Policy context}

While tackling obesity is high on the public health agenda both in the UK and internationally there is a lack of accessible information to inform policymakers and service commissioners of services about what types of interventions are most effective in reducing such inequalities in obesity. The Priority Public Health Conditions group (Task Group 8) of the Department of Health commissioned Strategic Review of Health Inequalities in England post-2010 (Marmot Review) [12,13] noted this evidence gap as did the EPPI Centre report on obesity (2008), which called for future systematic reviews to examine the effectiveness of interventions in reducing inequalities [14].

Internationally, there have been similar calls for research in this area. Robertson et al. identify the need for 'evidence of the reach and penetration of interventions in lower income groups' as a priority for research [7]. The World Health Organization (WHO) Commission on the Social Determinants of Health also highlighted the relative importance attached to the development and testing of social determinants of health indicators and intervention impact evaluation' [15]. Further, there is increasing recognition among policymakers that in order to effectively tackle complex health problems, such as obesity, and to reduce health inequalities, policy action across different intervention levels (including individual, community and society) as well as across the life course (childhood to adulthood) is required. Therefore, it is critical that evidence on the effectiveness of different types of interventions that tackle inequalities in obesity at these various levels are systematically identified, appraised and synthesized. To our knowledge there are no systematic reviews to date that have examined the effectiveness of interventions to reduce socioeconomic inequalities in obesity internationally.

The systematic review proposed here will address the aforementioned knowledge gap by reviewing primary studies that have considered the effectiveness of interventions that seek to reduce socioeconomic inequalities in obesity among adults. We approach this review in a whole systems way incorporating a multilevel perspective. This review will subsequently examine public health interventions at the individual, community and societal level $[4,8]$ that tackle inequalities in obesity. It will also examine organization, implementation and delivery of such interventions. This adult review complements our previous systematic review project of interventions to reduce inequalities in childhood obesity [16]. 
Tackling inequalities in obesity using a whole systems approach: developing an intervention framework

The Foresight review of obesity stressed the importance of a whole systems approach to tackling the 'obesity epidemic' [4]. Within this approach interventions are needed that target the wider social determinants of obesity [6]. Whitehead and Dahlgren's [17] oft-cited social model of health rainbow considers various levels of influence on health: individual (including biological and lifestyle factors), socio-cultural, living and working conditions and broader socioeconomic, cultural and environmental conditions. These are potential levels for intervention for reducing obesity. Using a whole systems approach and considering these various levels of intervention, we have developed an intervention framework (Table 1), which was used in our previous protocol for child obesity; however, we have updated it to reflect our focus on adult obesity in this protocol.

An overwhelming number of studies have focused on interventions at the individual level including educational, behavioral and pharmaceutical approaches. Swinburn et al. [18] argue that these types of interventions have only had a modest impact on reducing obesity and that there is a real need to focus on environments that are either 'obesogenic' (promotes obesity) or 'lepogenic' (promote leanness). They classify the environment into micro- and macro-environments. Micro-environments include how individuals interact with schools, workplaces, homes, and neighborhoods. Macro-environments include education, health systems, government, the food industry and societal attitudes and beliefs. Within these environments we may categorize them into physical, economic, political, or sociocultural.

The intervention framework proposed in this review considers these broader determinants of health in order to understand the mechanisms through which the different layers of intervention may operate to reduce socioeconomic inequalities in health. There are four levels of interventions to tackle inequalities: 1) strengthening individuals (person based strategies to improve the health of disadvantaged individuals); 2) strengthening communities (improving the health of disadvantaged communities and local areas by building social cohesion and mutual support); 3) improving living and school environments (reducing exposure to health-damaging material and psychosocial environments across the whole population); and 4) promoting healthy macro policy (improving the macro-economic, cultural and environmental context which influence the standard of living achieved by the whole population). According to Graham and Kelly, these interventions are underpinned by one of three different approaches to health inequality: 1) disadvantage (improving the absolute position of the most disadvantaged individuals and groups); 2) gap (reducing the

Table $1 \mathrm{~A}$ framework for tackling inequalities in obesity

\begin{tabular}{|c|c|c|c|c|c|}
\hline \multirow{3}{*}{\multicolumn{2}{|c|}{$\begin{array}{l}\text { APPROACH TO } \\
\text { TACKLING HEALTH } \\
\text { INEQUALTY }\end{array}$}} & \multicolumn{4}{|c|}{ LEVEL OF INTERVENTION } \\
\hline & & \multirow{2}{*}{$\begin{array}{c}\text { Individual } \\
\text { Strengthening individuals }\end{array}$} & \multirow{2}{*}{$\begin{array}{l}\text { Community } \\
\text { Strengthening communities }\end{array}$} & \multicolumn{2}{|c|}{ Societal } \\
\hline & & & & $\begin{array}{c}\text { Improving living and work } \\
\text { environment }\end{array}$ & $\begin{array}{c}\text { Promoting healthy macro } \\
\text { policies }\end{array}$ \\
\hline $\begin{array}{l}\text { Disadvantage } \\
\text { Gap }\end{array}$ & Targeted & $\begin{array}{l}\text { Health education, health } \\
\text { promotion and social } \\
\text { marketing; Diet and exercise } \\
\text { advice and counseling; } \\
\text { Weight management advice } \\
\text { and monitoring; Conditional } \\
\text { cash transfers; Lifestyle } \\
\text { counseling; Exercise on } \\
\text { prescription. }\end{array}$ & $\begin{array}{l}\text { Community health and } \\
\text { fitness centers; Health } \\
\text { trainers; Group, work or } \\
\text { community based exercise } \\
\text { programs; Group, work or } \\
\text { community diet, lifestyle, or } \\
\text { weight management advice } \\
\text { and/or counseling; Healthy } \\
\text { eating campaigns in } \\
\text { workplaces; Group or } \\
\text { community organized } \\
\text { education or support; } \\
\text { Localized point of sale social } \\
\text { marketing; Neighborhood } \\
\text { based physical activity } \\
\text { programs. }\end{array}$ & & \\
\hline Gradient & Universal & & & $\begin{array}{l}\text { Access to physical fitness } \\
\text { facilities (e.g. gym subsidies); } \\
\text { Availability of healthy food; } \\
\text { Green spaces, walk-ability } \\
\text { and the built environment; } \\
\text { Traffic light labeling. }\end{array}$ & $\begin{array}{l}\text { Restrictions on advertising } \\
\text { high fat and high sugar } \\
\text { foods; Food prices and } \\
\text { agricultural subsidies (e.g. } \\
\text { changing the Common } \\
\text { Agricultural Policy); Fiscal } \\
\text { measures to regulate supply } \\
\text { and demand (e.g. taxing } \\
\text { high fat and high sugar } \\
\text { foods). }\end{array}$ \\
\hline
\end{tabular}


relative gap between the best and worst off groups); or, 3) gradient (reducing the entire social gradient) [19]. Interventions are thus either targeted (such as individual level interventions underpinned by health as disadvantage) or universal (such as living and working conditions interventions that potentially influence the entire social gradient in health). In the proposed systematic review, the obesity interventions will be grouped according to this framework.

\section{Methods/Design}

The review will follow the same procedure carried out for the systematic review of the effectiveness of public health interventions at reducing socioeconomic inequalities in obesity among children [16]. The review will be carried out following established criteria for the good conduct and reporting of systematic reviews [20,21]. A Study Steering Group comprising key stakeholders from the UK policy and research communities, international representatives, a statistician and a health economist will guide the research. The review is registered with the PROSPERO International Prospective Register of Systematic Reviews (registration number: CRD42013003612).

\section{Objectives}

This project has two objectives:

1. To systematically review the effectiveness of public health interventions (individual, community and societal) in reducing socioeconomic inequalities in obesity among adults.

2. To establish how such public health interventions are organized, implemented and delivered.

\section{Interventions}

The review will examine public health interventions at the individual, community and societal level that might reduce inequalities in obesity among adults aged 18 years and over, in any setting, in any country. The review will utilize the intervention framework (Table 1) and group interventions as individual, community or societal with acknowledgement that some interventions might be cross-cutting. For example an individual level intervention could be a relatively complex weight management program providing dietary and physical activity education with behavioral counseling delivered individually (as opposed to group-based) or could simply be a gift card given to low-income families for the purchase of fruits and vegetables. An example of a community level intervention may be access to a community fitness center (with/without the addition of physical activity education), or a workplace health promotion campaign involving dietary and physical activity education. A societal level intervention may also be a workplace health promotion campaign but one that involves an environmental change such as healthy food choices in the work canteen. The review will consider public health strategies which might reduce existing inequalities in the prevalence of obesity as well as those interventions which might prevent the development of inequalities in obesity. However, clinical interventions such as those using drugs or surgery and laboratory-based studies will be excluded. We will also exclude studies of interventions designed for adults with a critical illness or severe comorbidities (such as diabetes, metabolic syndrome, hypertension and mental health disorders).

\section{Study designs}

A rigorous and inclusive international literature search will be conducted for all randomized and nonrandomized controlled trials, prospective and retrospective cohort studies (with/without control groups) and prospective repeat cross-sectional studies (with/without control groups) of the effectiveness of public health interventions at reducing inequalities in childhood obesity. Studies with a duration of at least 12 weeks (combination of intervention and follow up) will be included; an inclusion criterion used in previous Cochrane reviews of interventions aimed at preventing obesity in children [22] and of the effectiveness of exercise for weight loss in adults with overweight or obesity [23].

\section{Search strategy}

The search strategy (Additional file 1) will include the following electronic database searches (host sites given in parentheses) MEDLINE (Ovid), EMBASE (Ovid), CINAHL (EbscoHost), PsycINFO (EbscoHost), Social Science Citation Index (Web of Science), ASSIA (CSA), IBSS (EBSCO), Sociological Abstracts (CSA), and the NHS Economic Evaluation Database (NHS CRD). The skills of a trained information scientist (HJM) will be used to develop and implement the electronic searches. All databases will be searched from start date to present. We will not exclude papers on the basis of language, country or publication date.

We will supplement the electronic database searches with website and gray literature searches. We will hand search the bibliographies of all included studies and request relevant information on unpublished and in-progress research from key experts in the field. In addition, we will hand search the last two years of the most common five journals revealed by the electronic searches as well as journals identified by experts in the subject area.

\section{Outcomes}

In terms of outcomes, we will only include studies if they include a primary outcome that is a proxy for body 
fatness (weight and height, body mass index, waist measurement/waist to hip proportion, percentage fat content, skin fold thickness, and ponderal index in relation to childhood obesity). Data on related secondary outcomes (such as physical activity levels; dietary intake; blood results, such as cholesterol and glucose levels) will also be extracted from those studies which have a primary outcome. We will include both measured and selfreported outcomes. Studies will only be included if they have examined differential effects with regard to socioeconomic status (education, income, occupation, social class, deprivation, and poverty) or the intervention must have been targeted specifically at disadvantaged groups (for example, unemployed, low SES and low income) or deprived areas. Data on the organization, implementation and delivery of interventions will be extracted using existing methodological tools which assess the implementation of complex public health interventions [24], adapted and refined for the purposes of this review. Examples of the implementation components that will be examined include: theoretical underpinning; implementation context; experience of intervention team (planners and implementers); consultation/collaboration processes (planning and delivery stages); and resources (for example, time, money, staff, and equipment).

\section{Data extraction and quality appraisal}

The initial screening of titles and abstracts and full paper inclusion will be conducted by one reviewer (FCH or JMC) with a random $10 \%$ of the sample checked by a second reviewer $(\mathrm{HJM}$ or $\mathrm{FCH})$. Data extraction and methodological quality appraisal of the included studies will be conducted by one reviewer (FCH, JMC, CLB, or CDS) using established data extraction forms [20,25-30] and will be checked by a second reviewer (FCH, JMC, CLB, or CDS). Any discrepancies will be resolved through discussion between the authors and, if consensus is not reached, with the project lead (CLB). The methodological quality of the included studies will also be appraised using the Cochrane Public Health Review Group recommended Effective Public Health Practice Project Quality Assessment Tool for Quantitative Studies [31], which includes, among other things, an examination of sampling strategy, response and follow-up rates, intervention integrity, statistical analyses and assessment of adjustment for confounders. We will use the quality appraisal criteria for descriptive purposes and to highlight variations between studies.

\section{Analysis and synthesis}

Where possible, meta-analysis will be used to synthesize data using Comprehensive Meta-Analysis (Biostat, Englewood, NJ, USA) based on the primary outcomes. A fixed effect model will be used for the meta-analysis, unless there is evidence of heterogeneity between studies, in which case a random effect model will be used. The presence of heterogeneity will be investigated with the use of likelihood ratio test statistic, while funnel plot will be considered to explore publication bias. However, where meta-analysis is not possible, narrative synthesis will be conducted. We will report our analyses in accordance with PRISMA guidelines [32]. The main analysis will examine the effects of 1) individual, 2) community and 3) societal level public health interventions on socioeconomic inequalities in obesity, using the multi-dimensional framework outlined in Table 1. Where data permits, we will conduct demographic subgroup analysis by age, gender and ethnicity.

\section{Discussion}

The review will consider public health strategies that reduce existing inequalities in the prevalence of obesity as well as those interventions that might prevent the development of inequalities in obesity. The review will also serve as a mapping exercise of the types of interventions that have been evaluated in relation to tackling inequalities in obesity in adults, thereby highlighting any gaps in the evidence base. The review will also seek to establish how public health interventions that might reduce or prevent inequalities in obesity are organized, implemented and delivered. Context is increasingly recognized as an important factor in the success of public health interventions [33]. However, the assessment of implementation has not really featured strongly in previous obesity reviews. We will therefore develop, refine and apply existing methodological tools that assess the implementation of complex public health interventions [24].

We anticipate that a sizeable amount of literature will be located for synthesis as a result of our extensive search strategy, along with gray literature and website searches, and inclusive study design criteria. The size of the available evidence base will also be extended because we are looking at different levels of intervention: individual, community and societal, and we will evaluate interventions that are targeted at deprived groups or areas as well as those that include comparative data on the effects of interventions on differential impacts across two or more socioeconomic groups. The study design inclusion criteria in the review are broad given that while trials of individual-, and even community-level interventions are likely, we expect a dearth of experimental studies in relation to societal-level interventions as societal-level interventions tend not to be easily evaluated using experimental study designs [8]. Further, other recent systematic reviews of the effects of societal level public health interventions on socioeconomic inequalities in health have located few relevant experimental studies [34]. Full papers of all studies that fit our population, intervention, design and health outcome inclusion criteria will be examined, 
even if there is no mention of socioeconomic inequalities in the abstract; in this manner we will reduce the possibility of excluding studies that undertook subgroup analyses by socioeconomic status but did not publish the findings in the abstract. These strategies will increase the comprehensiveness of the search strategy and, therefore, the quality of the final synthesis.

We plan to hold an 'implications for policy and practice' review dissemination workshop with invited NHS commissioners with responsibilities for obesity; Department of Health policy makers with responsibility for obesity and inequalities; user group representatives (for example, community groups, schools and employer organizations, trade union congress); along with UK research network representatives (for example, Faculty of Public Health, Nutrition Society, UKPHA) to discuss the results, aid write up and facilitate translation of the findings into practice. Once the technical report and executive summary are finalized a short 'key findings' summary of the research will be sent to relevant stakeholders. The research will be disseminated via national and international academic/practitioner cross-over conferences, and a policy orientated summary paper will be published on an open access basis so that it is freely available to practitioners and the public.

\section{Additional file}

Additional file 1: Search Strategy - MEDLINE (Ovid) 1946 to 10 October 2012.

\section{Competing interests}

The authors declare that they have no competing interests.

\section{Authors' contributions}

CLB is Principal Investigator and led the writing of the manuscript. FCH is project manager and co-investigator and contributed to the writing and revision of the manuscript. HJM, JMC and CDS are co-investigators and contributed to the writing and revision of the manuscript. All authors read and approved the final manuscript.

\section{Acknowledgements}

This project is funded from a grant by the National Institute for Health Research Public Health Research Programme (grant 09/3010/14) and is registered with the PROSPERO International Prospective Register of Systematic Reviews (registration number: CRD42013003612, Available from http://www.crd.york.ac.uk/PROSPERO/ display_record.asp?ID=CRD42013003612). We would like to thank our steering group members for their comments: Goof Buijs (Netherlands Institute for Health Promotion and Disease Prevention), Richard Cookson (University of York), Adetayo Kasim (Durham University), Mike Kelly (National Institute for Health and Clinical Excellence), Louise Potvin (University of Montreal) and Martin White (Newcastle University). We would also like to thank Kerry Joyce (Newcastle University) for her input in to the preliminary research application.

The views and opinions expressed therein are those of the authors and do not necessarily reflect those of the Steering Group, NIHR PHR programme or the Department of Health.

\section{Author details}

${ }^{1}$ Department of Geography, Wolfson Research Institute, Durham University Queen's Campus, University Boulevard, Stockton-on-Tees TS17 6BH, UK. ${ }^{2}$ School of Medicine, Pharmacy and Health, Wolfson Research Institute, Durham University Queen's Campus, University Boulevard, Stockton-on-Tees TS17 6BH, UK
Received: 18 January 2013 Accepted: 26 April 2013

Published: 10 May 2013

\section{References}

1. World Health Organisation: Obesity: preventing and managing a global epidemic: Report of a WHO consultation of Obesity. Geneva: World Health Organisation Technical Report Series; 2000:894.

2. Cross-Government Obesity Unit: Department of Health, Department of Children Schools and Families: Healthy Weight, Healthy Lives: A Cross-Government Strategy for England. London: The Stationary Office; 2008

3. OECD: Obesity and the Economics of Prevention: Fit not Fat. Paris: OECD Publishing; 2010.

4. Butland B, Jebb S, Kopelman P: McPherson K, Thomas S, Mardell J, Parry V: Tackling obesities: Future choices - Project Report. London: Government Office for Science; 2007.

5. Health and Social Care Information Centre: Statistics on obesity, physical activity and diet: England, February 2009. Leeds; 2009.

6. Friel S, Chopra M, Satcher D: Unequal weight: equity oriented policy responses to the global obesity epidemic. BMJ 2007, 335:1241-1243.

7. Robertson A, Lobstein T, Knai C: Obesity and socio-economic groups in Europe: Evidence review and implications for action. Report SANCO/2005/C4-NUTRITION-03. Brussels: European Commission; 2007.

8. Law C, Power C, Graham H, Merrick D: Obesity and health inequalities. Obes Rev 2007, 8:19-22.

9. White M, Adamson A, Chadwick T, Dezateux C, Griffiths L, Howel D, Kelly S, Law C, Li L, Lo Conte R, Power C, Stamp E: The changing social patterning of obesity: an analysis to inform practice and policy development. Final report to the Policy Research Programme. London: Department of Health; 2007.

10. Mackenbach JP, Stirbu I, Roskam A-JR, Schaap MM, Menvielle G, Leinsalu M, Kunst AE: Socioeconomic inequalities in health in 22 European countries. N Engl J Med 2008, 358:2468-2481.

11. van Lenthe FJ, Mackenbach JP: Neighbourhood deprivation and overweight: the GLOBE study. Int J Obes 2002, 26:234-240.

12. Bambra C, Joyce K, Maryon-Davies A: Strategic Review of Health Inequalities in England Post-2010 (Marmot Review) (Task Group 8: Priority public health conditions: final report). In Book Strategic Review of Health Inequalities in England Post-2010 (Marmot Review) (Task Group 8: Priority public health conditions: final report. London: University College London; 2009.

13. Bambra C, Joyce KE, Bellis MA, Greatley A, Greengross S, Hughes S, Lincoln P, Lobstein T, Naylor C, Salay R, Wiseman M, Maryon-Davis A: Reducing health inequalities in priority public health conditions: using rapid review to develop proposals for evidence-based policy. J Public Health 2010, 32:496-505.

14. Woodman J, Lorenc T, Harden A, Oakley A: Social and environmental interventions to reduce childhood obesity: a systematic map of reviews. EPPI-Centre Report No. 1610. London: EPPI-Centre, Social Science Research Unit, Institute of Education, University of London; 2008.

15. WHO Commission on the Social Determinants of Health: Closing the gap in a generation: health equity through action on the social determinants of health. Final Report of the Commission on Social Determinants of Health Geneva: World Health Organisation; 2008.

16. Bambra C, Hillier F, Moore H, Summerbell C: Tackling inequalities in obesity: a protocol for a systematic review of the effectiveness of public health interventions at reducing socioeconomic inequalities in obesity amongst children. Syst Rev 2012, 1:16.

17. Whitehead M, Dahlgren $\mathrm{G}$ : What can be done about inequalities in health? Lancet 1991, 338:1059-1063.

18. Swinburn B, Egger G, Raza F: Dissecting obesogenic environments: the development and application of a framework for identifying and prioritizing environmental interventions for obesity. Prev Med 1999, 29:563-570.

19. Graham H, Kelly M: Health inequalities: concepts, frameworks and policy. London: Health Development Agency; 2004.

20. Centre for Reviews and Dissemination: Undertaking systematic reviews of research on effectiveness. CRD's guidance for carrying out or commissioning reviews 2nd edition. York: University of York; 2001

21. Higgins J, Green S: Cochrane Handbook for Systematic Reviews of Interventions Version 5.1.0 [updated March 2011]. The Cochrane Collaboration; 2011. Available from www.cochrane-handbook.org.

22. Summerbell C, Waters E, Edmunds L, Kelly S, Brown T, Campbell K: Interventions for preventing obesity in children. Cochrane Database Syst Rev 2008, 3:CD001871. 
23. Shaw K, Gennat H, O'Rourke P, Del Mar C: Exercise for overweight or obesity. Cochrane Database Syst Rev 2006, 4:CD003817.

24. Egan M, Bambra C, Petticrew M, Whitehead M: Reviewing evidence on complex social interventions: appraising implementation in systematic reviews of the health effects of organisational-level workplace interventions. J Epidemiol Community Health 2009, 63:4-11.

25. Bambra C, Egan M, Thomas S, Petticrew M, Whitehead M: The psychosocial and health effects of workplace reorganisation. 2. A systematic review of task restructuring interventions. J Epidemiol Community Health 2007, 61:1028-1037.

26. Bambra C, Whitehead M, Sowden A, Akers J, Petticrew M: A hard day's night? The effects of Compressed Working Week interventions on the health and work-life balance of shift workers: a systematic review. J Epidemiol Community Health 2008, 62:764-777.

27. Bambra CL, Whitehead MM, Sowden AJ, Akers J, Petticrew MP: Shifting Schedules: the health effects of reorganizing shift work. Am J Prev Med 2008, 34:427-434.

28. Deeks J, Dinnes J, D'Amico R, Sowden A, Sakarovitch C, Song F, Petticrew M, Altman D: Evaluating non-randomised intervention studies. Health Technol Assess 2003, 7:1-173.

29. Egan M, Bambra C, Thomas S, Petticrew M, Whitehead M, Thomson H: The psychosocial and health effects of workplace reorganisation 1: a systematic review of interventions that aim to increase employee participation or control. J Epidemiol Community Health 2007, 61:945-954.

30. Joyce K, Pabayo R, Critchley J, Bambra C: Flexible working conditions and their effects on employee health and wellbeing. Cochrane Database Syst Rev 2010, 2:CD008009.

31. Effective Public Health Practice Project Quality Assessment Tool for Quantitative Studies. http://www.ephpp.ca/tools.html.

32. Liberati A, Altman DG, Tetzlaff J, Mulrow C, Gøtzsche PC, loannidis JP, Clarke M, Devereaux PJ, Kleijnen J, Moher D: The PRISMA statement for reporting systematic reviews and meta-analyses of studies that evaluate healthcare interventions: explanation and elaboration. BMJ 2009, 339:b2700.

33. Chow CK, Lock K, Teo K, Subramanian S, McKee M, Yusuf S: Environmental and societal influences acting on cardiovascular risk factors and disease at a population level: a review. Int J Epidemiol 2009, 38:1580-1594.

34. Bambra C, Gibson M, Sowden A, Wright K, Whitehead M, Petticrew M: Tackling the wider social determinants of health and health inequalities: evidence from systematic reviews. J Epidemiol Community Health 2010, 64:284-291.

doi:10.1186/2046-4053-2-27

Cite this article as: Bambra et al:: Tackling inequalities in obesity: a protocol for a systematic review of the effectiveness of public health interventions at reducing socioeconomic inequalities in obesity among adults. Systematic Reviews 2013 2:27.

\section{Submit your next manuscript to BioMed Central and take full advantage of:}

- Convenient online submission

- Thorough peer review

- No space constraints or color figure charges

- Immediate publication on acceptance

- Inclusion in PubMed, CAS, Scopus and Google Scholar

- Research which is freely available for redistribution 